\title{
Weighted Hardy type inequalities for supremum operators on the cones of monotone functions
}

\author{
Lars-Erik Persson ${ }^{1,2^{*}}$, Guldarya E Shambilova ${ }^{3}$ and Vladimir D Stepanov ${ }^{4}$
}

\section{"Correspondence:}

Lars-Erik.Persson@ltu.se

'Department of Engineering

Sciences and Mathematics, Lulea

University of Technology, Lulea, 97187, Sweden

${ }^{2} \mathrm{UiT}$, The Arctic University of Norway, P.O. Box 385, Narvik, 8505, Norway

Full list of author information is available at the end of the article

\begin{abstract}
The complete characterization of the weighted $L^{p}-L^{r}$ inequalities of supremum operators on the cones of monotone functions for all $0<p, r \leq \infty$ is given.
\end{abstract}

MSC: Primary 26D15; secondary 47G10

Keywords: Hardy type inequality; weight; supremum operator; Lebesgue space; monotone function

\section{Introduction}

Let $\mathbb{R}_{+}:=[0, \infty)$. Denote $\mathfrak{M}$ the set of all measurable functions on $\mathbb{R}_{+}, \mathfrak{M}^{+} \subset \mathfrak{M}$ the subset of all non-negative functions and $\mathfrak{M}^{\downarrow} \subset \mathfrak{M}^{+}\left(\mathfrak{M}^{\uparrow} \subset \mathfrak{M}^{+}\right)$is the cone of all non-increasing (non-decreasing) functions. Also denote by $\mathscr{C} \subset \mathfrak{M}$ the set of all continuous functions on $\mathbb{R}_{+}$. If $0<p \leq \infty$ and $v \in \mathfrak{M}^{+}$we define

$$
\begin{aligned}
& L_{v}^{p}:=\left\{f \in \mathfrak{M}:\|f\|_{L_{v}^{p}}:=\left(\int_{0}^{\infty}|f(x)|^{p} v(x) d x\right)^{\frac{1}{p}}<\infty\right\}, \\
& L_{v}^{\infty}:=\left\{f \in \mathfrak{M}:\|f\|_{L_{v}^{\infty}}:=\underset{x \geq 0}{\operatorname{ess} \sup } v(x)|f(x)|<\infty\right\} .
\end{aligned}
$$

Let $w \in \mathfrak{M}^{+}$and $k(x, y) \geq 0$ is a Borel function on $[0, \infty)^{2}$ satisfying Oinarov's condition: $k(x, y)=0$ if $x<y$, and there is a constant $D \geq 1$ independent of $x \geq z \geq y \geq 0$ such that

$$
\frac{1}{D}(k(x, z)+k(z, y)) \leq k(x, y) \leq D(k(x, z)+k(z, y)) .
$$

The mapping properties between weighted $L^{p}$ spaces of Hardy type operators involved are very well studied. See e.g. the books [1, 2] and [3] and the references therein. We also mention the following examples of articles in this area: [4-8] and [9]. Recently, it has been discovered that it is of great interest to study also some corresponding supremum operators instead of the usual such Hardy type (arithmetic mean) operators. The interest comes both from purely mathematical point of view but also from various applications where such kernels many times are the unit impulse answers to the problem at hand and

(C) 2016 Persson et al. This article is distributed under the terms of the Creative Commons Attribution 4.0 International License (http://creativecommons.org/licenses/by/4.0/), which permits unrestricted use, distribution, and reproduction in any medium, provided you give appropriate credit to the original author(s) and the source, provide a link to the Creative Commons license, and indicate if changes were made. 
the best constants means the operator norms of the corresponding transfer of the energy of the 'signals' measured in weighted $L^{p}$ spaces.

We consider supremum operators of the form

$$
\begin{aligned}
& (T f)(x)=\underset{y \geq x}{\operatorname{ess} \sup } k(y, x) w(y) f(y), \quad f \in \mathfrak{M}^{\uparrow}, \\
& (S f)(x)=\underset{y \geq x}{\operatorname{ess} \sup } k(y, x) w(y) f(y), \quad f \in \mathfrak{M}^{\downarrow}, \\
& (\mathscr{T} f)(x)=\underset{0 \leq y \leq x}{\operatorname{ess} \sup } k(x, y) w(y) f(y), \quad f \in \mathfrak{M}^{\downarrow}, \\
& (\mathscr{S} f)(x)=\underset{0 \leq y \leq x}{\operatorname{ess} \sup } k(x, y) w(y) f(y), \quad f \in \mathfrak{M}^{\uparrow} .
\end{aligned}
$$

Let $0<p, r \leq \infty$ and $u, v \in \mathfrak{M}^{+}$. The paper is devoted to the necessary and sufficient conditions for the inequalities

$$
\begin{aligned}
& \|T f\|_{L_{u}^{r}} \leq C_{T}\|f\|_{L_{v}^{p}}, \quad f \in \mathfrak{M}^{\uparrow}, \\
& \|S f\|_{L_{u}^{r}} \leq C_{S}\|f\|_{L_{v}^{p}}, \quad f \in \mathfrak{M}^{\downarrow}, \\
& \|\mathscr{T} f\|_{L_{u}^{r}} \leq C_{\mathscr{T}}\|f\|_{L_{v}^{p}}, \quad f \in \mathfrak{M}^{\downarrow}, \\
& \|\mathscr{S} f\|_{L_{u}^{r}} \leq C_{\mathscr{S}}\|f\|_{L_{v}^{p}}, \quad f \in \mathfrak{M}^{\uparrow},
\end{aligned}
$$

where the constants $C_{T}$ and others are taken as the least possible.

This problem was first studied for the inequality (1.3) in [10], Theorem 3.2, in a case when $k(x, y)=1, w \in \mathscr{C}$. This result was extended in [11] for the case $k(x, y)$ satisfying (1.1) with a discrete form of a criterion for $0<r<p<\infty$. With different supremum operators some similar problems were studied in [12-22]. This area is currently developing intensively and finds many interesting applications.

Section 2 is devoted to preliminaries. The border cases $0<r<p=\infty, 0<p<r=\infty$ and $r=p=\infty$ are solved in Section 3. In Section 4 we characterize the case $k(x, y)=1$, which is essentially used in Section 5 with the main results of the paper.

We use signs := and $=$ : for determining new quantities and $\mathbb{Z}$ for the set of all integers. For positive functionals $F$ and $G$ we write $F \lesssim G$, if $F \leq c G$ with some positive constant $c$, which depends only on irrelevant parameters. $F \approx G$ means $F \lesssim G \lesssim F$ or $F=c G$. $\chi_{E}$ denotes the characteristic function (indicator) of a set $E$. Uncertainties of the form $0 \cdot \infty, \frac{\infty}{\infty}$ and $\frac{0}{0}$ are taken to be zero. $\square$ stands for the end of a proof.

\section{Preliminaries}

We denote

$$
V(t):=\int_{0}^{t} v, \quad V_{*}(t):=\int_{t}^{\infty} v .
$$

Let $0<p, r<\infty$. By [11], Lemma 2.1, and the monotone convergence theorem the inequality (1.2) is equivalent to

$$
\left(\int_{0}^{\infty}\left[\underset{y \geq x}{\operatorname{ess} \sup }(k(y, x) w(y))^{p} \int_{0}^{y} h\right]^{\frac{r}{p}} u(x) d x\right)^{\frac{p}{r}} \leq C_{T}^{p} \int_{0}^{\infty} h V_{*}, \quad h \in \mathfrak{M}^{+} .
$$


If

$$
T_{p} h(x):=\underset{y \geq x}{\operatorname{ess} \sup }(k(y, x) w(y))^{p} \int_{0}^{y} h,
$$

then (2.1) is equivalent to

$$
\left\|T_{p} h\right\|_{L_{u}^{\frac{r}{p}}} \leq C_{T}^{p}\|h\|_{L_{V_{*}}^{1}}, \quad h \in \mathfrak{M}^{+} .
$$

Analogously, if

$$
\begin{aligned}
& S_{p} h(x):=\underset{y \geq x}{\operatorname{ess} \sup }(k(y, x) w(y))^{p} \int_{y}^{\infty} h, \\
& \mathscr{T}_{p} h(x):=\underset{0 \leq y \leq x}{\operatorname{ess} \sup }(k(x, y) w(y))^{p} \int_{y}^{\infty} h, \\
& \mathscr{S}_{p} h(x):=\underset{0 \leq y \leq x}{\operatorname{ess} \sup }(k(x, y) w(y))^{p} \int_{0}^{y} h,
\end{aligned}
$$

then (1.3), (1.4), and (1.5) are equivalent to

$$
\begin{aligned}
& \left\|S_{p} h\right\|_{L_{u}^{\frac{r}{p}}} \leq C_{S}^{p}\|h\|_{L_{V}^{1}}, \quad h \in \mathfrak{M}^{+}, \\
& \left\|\mathscr{T}_{p} h\right\|_{L_{u}^{\frac{r}{p}}} \leq C_{\mathscr{T}}^{p}\|h\|_{L_{V}^{1}}, \quad h \in \mathfrak{M}^{+},
\end{aligned}
$$

and

$$
\left\|\mathscr{S}_{p} h\right\|_{L_{u}^{\frac{r}{p}}} \leq C_{\mathscr{S}}^{p}\|h\|_{L_{V_{*}}^{1}}, \quad h \in \mathfrak{M}^{+}
$$

respectively.

For the border cases $0<p<r=\infty, 0<r<p=\infty$, and $r=p=\infty$ we have the following four groups of inequalities:

$$
\begin{aligned}
& \underset{x \geq 0}{\operatorname{ess} \sup }[u(x)]^{p} T_{p} h(x) \leq C_{T, p}^{p} \int_{0}^{\infty} h V_{*}, \quad h \in \mathfrak{M}^{+}, \\
& \left(\int_{0}^{\infty}[\underset{y \geq x}{\operatorname{ess} \sup } k(y, x) w(y) f(y)]^{r} u(x) d x\right)^{\frac{1}{r}} \leq C_{T, r}\|f\|_{L_{v}^{\infty}}, \quad f \in \mathfrak{M}^{\uparrow}, \\
& \underset{x \geq 0}{\operatorname{ess} \sup } u(x)[\underset{y \geq x}{\operatorname{ess} \sup } k(y, x) w(y) f(y)] \leq C_{T, \infty}\|f\|_{L_{v}^{\infty}}, \quad f \in \mathfrak{M}^{\uparrow},
\end{aligned}
$$

for the operator $T$;

$$
\begin{aligned}
& \underset{x \geq 0}{\operatorname{ess} \sup }[u(x)]^{p} S_{p} h(x) \leq C_{S, p}^{p} \int_{0}^{\infty} h V, \quad h \in \mathfrak{M}^{+}, \\
& \left(\int_{0}^{\infty}[\underset{y \geq x}{\operatorname{ess} \sup } k(y, x) w(y) f(y)]^{r} u(x) d x\right)^{\frac{1}{r}} \leq C_{S, r}\|f\|_{L_{v}^{\infty}}, \quad f \in \mathfrak{M}^{\downarrow}, \\
& \underset{x \geq 0}{\operatorname{ess} \sup u(x)}\left[\underset{y \geq x}{\operatorname{ess} \sup k(y, x) w(y) f(y)] \leq C_{S, \infty}\|f\|_{L_{v}^{\infty}}, \quad f \in \mathfrak{M}^{\downarrow},}\right.
\end{aligned}
$$


for the operator $S$;

$$
\begin{aligned}
& \underset{x \geq 0}{\operatorname{ess} \sup }[u(x)]^{p} \mathscr{T}_{p} h(x) \leq C_{\mathscr{T}, p}^{p} \int_{0}^{\infty} h V, \quad h \in \mathfrak{M}^{+}, \\
& \left(\int_{0}^{\infty}[\underset{0 \leq y \leq x}{\operatorname{ess} \sup } k(x, y) w(y) f(y)]^{r} u(x) d x\right)^{\frac{1}{r}} \leq C_{\mathscr{T}, r}\|f\|_{L_{v}^{\infty}}, \quad f \in \mathfrak{M}^{\downarrow}, \\
& \underset{x \geq 0}{\operatorname{ess} \sup u(x)}[\underset{0 \leq y \leq x}{\operatorname{ess} \sup } k(x, y) w(y) f(y)] \leq C_{\mathscr{T}, \infty}\|f\|_{L_{v}^{\infty}}, \quad f \in \mathfrak{M}^{\downarrow},
\end{aligned}
$$

for the operator $\mathscr{T}$, and

$$
\begin{aligned}
& \underset{x \geq 0}{\operatorname{ess} \sup }[u(x)]^{p} \mathscr{S}_{p} h(x) \leq C_{\mathscr{S}, p}^{p} \int_{0}^{\infty} h V_{*}, \quad h \in \mathfrak{M}^{+}, \\
& \left(\int_{0}^{\infty}[\underset{0 \leq y \leq x}{\operatorname{ess} \sup } k(x, y) w(y) f(y)]^{r} u(x) d x\right)^{\frac{1}{r}} \leq C_{\mathscr{S}, r}\|f\|_{L_{v}^{\infty}}, \quad f \in \mathfrak{M}^{\uparrow}, \\
& \underset{x \geq 0}{\operatorname{ess} \sup u(x)}[\underset{0 \leq y \leq x}{\operatorname{ess} \sup } k(x, y) w(y) f(y)] \leq C_{\mathscr{S}, \infty}\|f\|_{L_{v}^{\infty}}, \quad f \in \mathfrak{M}^{\uparrow},
\end{aligned}
$$

for the operator $\mathscr{S}$. We characterize the inequalities (2.6)-(2.17) in the next section.

To deal with the inequalities (2.1)-(2.5) we study first the case $k(x, y)=1$ and then a general case.

\section{Border cases of summation parameters}

For a measurable function $v \in \mathfrak{M}^{+}$we define monotone envelopes (see [23], Section 2) as follows:

$$
\begin{aligned}
& v^{\downarrow}(x):=\underset{y \geq x}{\operatorname{esssup}} v(y), \\
& v^{\uparrow}(x):=\underset{0 \leq y \leq x}{\operatorname{ess} \sup } v(y) .
\end{aligned}
$$

Theorem 3.1 For the best possible constants of the inequalities (2.6)-(2.8) we have

$$
\begin{aligned}
C_{T, p} & \approx \sup _{x \geq 0} u^{\uparrow}(x) \underset{y \geq x}{\operatorname{ess} \sup } \frac{k(y, x) w(y)}{V_{*}^{1 / p}(y)}, \\
C_{T, r} & =\left(\int_{0}^{\infty}\left[\underset{y \geq x}{\operatorname{ess} \sup } \frac{k(y, x) w(y)}{v^{\downarrow}(y)}\right]^{r} u(x) d x\right)^{\frac{1}{r}}, \\
C_{T, \infty} & =\underset{x \geq 0}{\operatorname{ess} \sup u(x)}\left[\underset{y \geq x}{\operatorname{ess} \sup } \frac{k(y, x) w(y)}{v^{\downarrow}(y)}\right] .
\end{aligned}
$$

Proof Observe that if $k(x, y)$ satisfies (1.1), then $[k(x, y)]^{p}$ satisfies (1.1) too with a constant $D_{p} \geq 1$. If $x \leq t$, then

$$
\begin{aligned}
T_{p} h(t) & =\underset{y \geq t}{\operatorname{ess} \sup }(k(y, t) w(y))^{p} \int_{0}^{y} h \\
& \leq D_{p} \underset{y \geq t}{\operatorname{ess} \sup }(k(y, x) w(y))^{p} \int_{0}^{y} h \\
& \leq D_{p} \underset{y \geq x}{\operatorname{ess} \sup }(k(y, x) w(y))^{p} \int_{0}^{y} h=D_{p} T_{p} h(x) .
\end{aligned}
$$


Hence,

$$
T_{p} h(x) \approx \sup _{t \geq x} T_{p} h(t):=\varphi(x) \in \mathfrak{M}^{\downarrow}
$$

It implies (see [11], Proposition 3.1)

$$
\begin{aligned}
\underset{x \geq 0}{\operatorname{ess} \sup }[u(x)]^{p} T_{p} h(x) & \approx \underset{x \geq 0}{\operatorname{ess} \sup }[u(x)]^{p} \varphi(x) \\
& =\underset{x \geq 0}{\operatorname{ess} \sup }[u(x)]^{p} \sup _{t \geq x} \varphi(t)=\sup _{t \geq 0} \varphi(t)\left[u^{\uparrow}(t)\right]^{p} \\
& \approx \sup _{x \geq 0}\left[u^{\uparrow}(x)\right]^{p} T_{p} h(x),
\end{aligned}
$$

and (2.6) is equivalent to

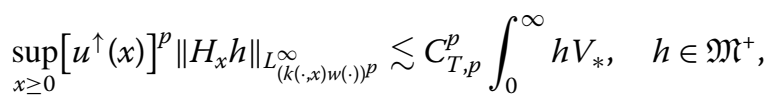

where

$$
H_{x} h(y):=\chi_{[x, \infty)}(y) \int_{0}^{y} h
$$

Thus,

$$
C_{T, p}^{p} \approx \sup _{x \geq 0}\left[u^{\uparrow}(x)\right]^{p}\left\|H_{x}\right\|_{L_{V_{*}}^{1} \rightarrow L_{(k(\cdot, x) w(\cdot))^{p}}^{\infty}}
$$

Since by a well-known theorem ([24], Theorem 1.1)

$$
\left\|H_{x}\right\|_{L_{V_{*}}^{1} \rightarrow L_{(k(\cdot, x) w(\cdot))^{p}}^{\infty}}=\operatorname{ess} \sup _{y \geq x} \frac{(k(y, x) w(y))^{p}}{V_{*}(y)}
$$

we obtain (3.1).

Now, (2.7) is equivalent to the inequality

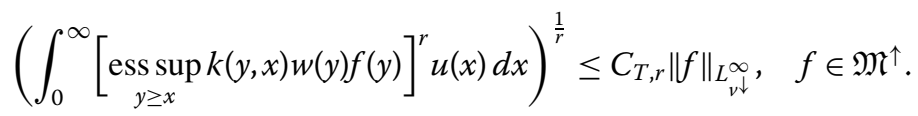

The lower bound of (3.2) follows from (3.5) with $f=\frac{1}{v \downarrow}$ and the upper bound from the estimate $f(y) \leq \frac{\|f\|_{L}^{\infty}}{v^{\downarrow}(y)}$

The proof of (3.3) is the same.

Analogously, we can prove the following.

Theorem 3.2 For the best possible constants of the inequalities (2.9)-(2.17) we have

$$
C_{S, p} \approx \sup _{x \geq 0} u^{\uparrow}(x) \operatorname{ess~sup} \frac{k(y, x) w(y)}{V^{1 / p}(y)}
$$




$$
\begin{aligned}
& C_{S, r}=\left(\int_{0}^{\infty}\left[\underset{y \geq x}{\operatorname{ess} \sup } \frac{k(y, x) w(y)}{v^{\uparrow}(y)}\right]^{r} u(x) d x\right)^{\frac{1}{r}}, \\
& C_{S, \infty}=\underset{x \geq 0}{\operatorname{ess} \sup } u(x)\left[\operatorname{ess\operatorname {sup}} \frac{k(y, x) w(y)}{v^{\uparrow}(y)}\right] \text {. } \\
& C_{\mathscr{T}, p} \approx \sup _{x \geq 0} u^{\downarrow}(x) \underset{0 \leq y \leq x}{\operatorname{ess} \sup } \frac{k(x, y) w(y)}{V^{1 / p}(y)}, \\
& C_{\mathscr{T}, r}=\left(\int_{0}^{\infty}\left[\underset{0 \leq y \leq x}{\operatorname{ess} \sup } \frac{k(x, y) w(y)}{v^{\uparrow}(y)}\right]^{r} u(x) d x\right)^{\frac{1}{r}}, \\
& C_{\mathscr{T}, \infty}=\underset{x \geq 0}{\operatorname{ess} \sup } u(x)\left[\operatorname{ess~sup}_{0 \leq y \leq x} \frac{k(x, y) w(y)}{v^{\uparrow}(y)}\right], \\
& C_{\mathscr{S}, p} \approx \sup _{x \geq 0} u^{\downarrow}(x) \operatorname{esssup}_{0 \leq y \leq x} \frac{k(y, x) w(y)}{V_{*}^{1 / p}(y)}, \\
& C_{\mathscr{S}, r}=\left(\int_{0}^{\infty}\left[\underset{0 \leq y \leq x}{\operatorname{ess} \sup } \frac{k(y, x) w(y)}{v^{\downarrow}(y)}\right]^{r} u(x) d x\right)^{\frac{1}{r}}, \\
& C_{\mathscr{S}, \infty}=\underset{x \geq 0}{\operatorname{ess} \sup } u(x)\left[\operatorname{ess~sup}_{0 \leq y \leq x} \frac{k(y, x) w(y)}{\nu^{\downarrow}(y)}\right] .
\end{aligned}
$$

\section{The case $k(x, y)=1$}

Let $u, v_{0}, w_{0} \in \mathfrak{M}^{+}$be weights. We suppose for simplicity that $0<\int_{0}^{t} u<\infty$, for all $t>0$, $\int_{0}^{\infty} u=\infty$ and define the functions $\sigma:[0 ; \infty) \rightarrow[0 ; \infty), \sigma^{-1}:[0 ; \infty) \rightarrow[0 ; \infty)$, by

$$
\begin{aligned}
& \sigma(x):=\inf \left\{y>0: \int_{0}^{y} u \geq 2 \int_{0}^{x} u\right\}, \\
& \sigma^{-1}(x):=\inf \left\{y>0: \int_{0}^{y} u \geq \frac{1}{2} \int_{0}^{x} u\right\} .
\end{aligned}
$$

Let $\sigma^{2}:=\sigma(\sigma)$. For $0 \leq c<d<\infty$ and $h \in \mathfrak{M}^{+}$we put

$$
\begin{aligned}
& H_{c} h(x):=\chi_{[c, \infty)}(x) \int_{0}^{x} h, \\
& H_{c, d} h(x):=\chi_{[c, d)}(x) \int_{\sigma^{-1}(c)}^{x} h, \\
& H_{c}^{*} h(x):=\chi_{[c, \infty)}(x) \int_{x}^{\infty} h, \\
& H_{c, d}^{*} h(x):=\chi_{[c, d)}(x) \int_{x}^{\sigma(d)} h .
\end{aligned}
$$

We need the following partial cases of [21], Theorems 2.1 and 2.3 (see also [19, 20]).

Theorem 4.1 Let $0<r<\infty$. Then:

(a) For validity of the inequality

$$
\left(\int_{0}^{\infty}\left[\underset{y \geq x}{\operatorname{ess} \sup } w_{0}(y) \int_{0}^{y} h\right]^{r} u(x) d x\right)^{\frac{1}{r}} \leq C_{0}\|h\|_{L_{v_{0}}^{1}}, \quad h \in \mathfrak{M}^{+},
$$


it is necessary and sufficient that the inequality

$$
\left(\int_{0}^{\infty} u(x)\left[w_{0}^{\downarrow}(x)\right]^{r}\left(\int_{0}^{x} h\right)^{r} d x\right)^{\frac{1}{r}} \leq A_{0}\|h\|_{L_{v_{0}}^{1}}, \quad h \in \mathfrak{M}^{+}
$$

holds and the constant

$$
A_{1}:= \begin{cases}\sup _{t>0}\left(\int_{0}^{t} u\right)^{\frac{1}{r}}\left\|H_{t}\right\|_{L_{v_{0}}^{1} \rightarrow L_{w_{0}}^{\infty},} \frac{r}{\frac{r}{1-r}} & r \geq 1, \\ \left(\int_{0}^{\infty} u(x)\left(\int_{0}^{x} u\right)^{\frac{r}{1-r}}\left\|H_{\left[\sigma^{-1}(x), \sigma(x)\right]}\right\|_{L_{v_{0}}^{1} \rightarrow L_{w_{0}}^{\infty}}^{\infty} d x\right)^{\frac{1-r}{r}}, & 0<r<1,\end{cases}
$$

is finite. Moreover, $C_{0} \approx A_{0}+A_{1}$.

(b) For validity of the inequality

$$
\left(\int_{0}^{\infty}\left[\underset{y \geq x}{\operatorname{ess} \sup } w_{0}(y) \int_{y}^{\infty} h\right]^{r} u(x) d x\right)^{\frac{1}{r}} \leq C_{1}\|h\|_{L_{\nu_{0}}^{1}}, \quad h \in \mathfrak{M}^{+}
$$

it is necessary and sufficient that the inequality

$$
\left(\int_{0}^{\infty} u(x)\left[\underset{x \leq y \leq \sigma^{2}(x)}{\operatorname{ess} \sup _{0}} w_{0}(y)\right]^{r}\left(\int_{\sigma^{2}(x)}^{\infty} h\right)^{r} d x\right)^{\frac{1}{r}} \leq B_{0}\|h\|_{L_{v_{0}}^{1}}, \quad h \in \mathfrak{M}^{+}
$$

holds and the constant

$$
B_{1}:= \begin{cases}\sup _{t>0}\left(\int_{0}^{t} u\right)^{\frac{1}{r}}\left\|H_{t}^{*}\right\|_{L_{v_{0}}^{1} \rightarrow L_{w_{0}}^{\infty},} & r \geq 1, \\ \left(\int_{0}^{\infty} u(x)\left(\int_{0}^{x} u\right)^{\frac{r}{1-r}}\left\|H_{\left[\sigma^{-1}(x), \sigma(x)\right]}^{*}\right\|_{L_{v_{0}}^{1} \rightarrow L_{w_{0}}^{\infty}}^{1-r} d x\right)^{\frac{r-r}{r}}, & 0<r<1,\end{cases}
$$

is finite. Moreover, $C_{1} \approx B_{0}+B_{1}$.

Using Theorem 4.1 we characterize (1.2) and (1.3) with $k(x, y)=1$.

Theorem 4.2 Let $0<p, r<\infty$ and $k(x, y)=1$. Then, for the best possible constants of the inequalities (1.2) and (1.3) the following equivalences hold:

$$
C_{T} \approx \mathscr{A}_{0}+\mathscr{A}_{1}, \quad C_{S} \approx \mathscr{B}_{0}+\mathscr{B}_{1},
$$

where

$$
\begin{aligned}
& \mathscr{A}_{0}=\sup _{t>0}\left[V_{*}(t)\right]^{-\frac{1}{p}}\left(\int_{t}^{\infty} u\left[w^{\downarrow}\right]^{r}\right)^{\frac{1}{r}}, \quad r \geq p, \\
& \mathscr{A}_{0}=\left(\int_{0}^{\infty}\left(\left[V_{*}(x)\right]^{-1} \int_{x}^{\infty} u\left[w^{\downarrow}\right]^{r}\right)^{\frac{r}{p-r}} u(x)\left[w^{\downarrow}(x)\right]^{r} d x\right)^{\frac{p-r}{p r}}, \quad 0<r<p, \\
& \mathscr{A}_{1}=\sup _{t>0}\left(\int_{0}^{t} u\right)^{\frac{1}{r}} \sup _{y \geq t} \frac{w^{\downarrow}(y)}{\left[V_{*}(y)\right]^{\frac{1}{p}}}, \quad r \geq p, \\
& \mathscr{A}_{1}=\left(\int_{0}^{\infty} u(x)\left(\int_{0}^{x} u\right)^{\frac{r}{p-r}}\left(\begin{array}{c}
\operatorname{ess} \sup \\
\sigma^{-1}(x) \leq y \leq \sigma(x)
\end{array} \frac{[w(y)]^{p}}{V_{*}(y)}\right)^{\frac{r}{p-r}} d x\right)^{\frac{p-r}{p r}}, \quad 0<r<p,
\end{aligned}
$$




$$
\begin{aligned}
\mathscr{B}_{0}= & \sup _{t>0}\left[V\left(\sigma^{2}(t)\right)\right]^{-\frac{1}{p}}\left(\int_{0}^{t} u(x)\left[\operatorname{ess~sup}_{x \leq y \leq \sigma^{2}(x)} w(y)\right]^{r} d x\right)^{\frac{1}{r}}, \quad r \geq p, \\
\mathscr{B}_{0}= & \left(\int_{0}^{\infty}\left(\left[V\left(\sigma^{2}(z)\right)\right]^{-1} \int_{0}^{z} u(x)\left[\operatorname{ess~sup}_{x \leq y \leq \sigma^{2}(x)} w(y)\right]^{r} d x\right)^{\frac{r}{p-r}}\right. \\
& \left.\times u(z)\left[\operatorname{ess~sup}_{z \leq y \leq \sigma^{2}(z)} w(y)\right]^{r} d z\right)^{\frac{p-r}{p r}}, \quad 0<r<p, \\
\mathscr{B}_{1}= & \sup _{t>0}\left(\int_{0}^{t} u\right)^{\frac{1}{r}} \operatorname{ess} \sup \frac{w(y)}{[V(y)]^{\frac{1}{p}}}, \quad r \geq p, \\
\mathscr{B}_{1}= & \left(\int_{0}^{\infty} u(x)\left(\int_{0}^{x} u\right)^{\frac{r}{p-r}}\left(\operatorname{esssup}_{\sigma^{-1}(x) \leq y \leq \sigma(x)} \frac{[w(y)]^{p}}{V(y)}\right)^{\frac{r}{p-r}} d x\right)^{\frac{p-r}{p r}}, \quad 0<r<p .
\end{aligned}
$$

Proof Since (1.2) $\Leftrightarrow$ (2.2) and (1.3) $\Leftrightarrow$ (2.3), the proof follows by applying Theorem 4.1 with $r$ replaced by $\frac{r}{p}, w_{0}=w^{p}, v_{0}=V_{*}$ in (4.1) and $v_{0}=V$ in (4.2). Thus, $C_{T} \approx \mathscr{A}_{0}^{\prime}+\mathscr{A}_{1}^{\prime}$, where $\mathscr{A}_{0}^{\prime}$ is the best constant in the inequality

$$
\left(\int_{0}^{\infty} u(x)\left[w^{\downarrow}(x)\right]^{r}\left(\int_{0}^{x} h\right)^{\frac{r}{p}} d x\right)^{\frac{p}{r}} \leq\left[\mathscr{A}_{0}^{\prime}\right]^{p}\|h\|_{L_{V_{*}}^{1}}, \quad h \in \mathfrak{M}^{+}
$$

and

$$
\left[\mathscr{A}_{1}^{\prime}\right]^{p}= \begin{cases}\sup _{t>0}\left(\int_{0}^{t} u\right)^{\frac{p}{r}}\left\|H_{t}\right\|_{L_{V_{*}}^{1} \rightarrow L_{w^{p}}^{\infty},} & r \geq p, \\ \left.\left(\int_{0}^{\infty} u(x)\left(\int_{0}^{x} u\right)^{\frac{r}{p-r}} \| H_{\left[\sigma^{-1}(x), \sigma(x)\right]}\right]_{L_{V_{*}}^{1} \rightarrow L_{w^{p}}^{p-r}}^{\infty} d x\right)^{\frac{p-r}{r}}, & 0<r<p .\end{cases}
$$

If $k(x, y) \geq 0$ is a measurable kernel on $\mathbb{R}_{+} \times \mathbb{R}_{+}$and

$$
K f(x):=\int_{0}^{\infty} k(x, y) f(y) d y
$$

then by well-known results ([25], Chapter XI, Section 1.5, Theorem 4, see also [24], Theorem 1.1)

$$
\|K\|_{L^{1} \rightarrow L^{q}}=\underset{s \geq 0}{\operatorname{ess} \sup }\|k(\cdot, s)\|_{L^{q}}, \quad 1 \leq q \leq \infty .
$$

If $k(x, y)=w(x) \chi_{[0, x]}(y) u(y)$ and $0<q<1$, then ([26], Theorem 3.3)

$$
\|K\|_{L^{1} \rightarrow L^{q}} \approx\left(\int_{0}^{\infty}[\underset{0 \leq y \leq x}{\operatorname{ess} \sup u(y)}]^{\frac{q}{1-q}}\left(\int_{x}^{\infty} w^{q}\right)^{\frac{q}{1-q}}[w(x)]^{q} d x\right)^{\frac{1-q}{q}} .
$$

Applying (4.5) and (4.6) to (4.4) we find that $\mathscr{A}_{0} \approx \mathscr{A}_{0}^{\prime}$. Again, applying (4.5), when

$$
k(x, y)=w^{p}(x) \chi_{[t, \infty)}(x) \frac{\chi_{[0, x]}(y)}{V_{*}(y)}
$$


we obtain

$$
\begin{aligned}
\left\|H_{t}\right\|_{L_{V_{*}}^{1} \rightarrow L_{w^{p}}^{\infty}} & =\underset{s \geq 0}{\operatorname{ess} \sup }\|k(\cdot, s)\|_{L^{\infty}}=\underset{s \geq 0}{\operatorname{ess} \sup } \frac{1}{V_{*}(s)} \underset{\{x \geq t\} \cap\{x \geq s\}}{\operatorname{ess} \sup } w^{p}(x) \\
& =\sup _{s \geq 0} \frac{1}{V_{*}(s)}\left[w^{\downarrow}(\max (t, s))\right]^{p} \\
& =\max \left(\sup _{0 \leq s \leq t} \frac{\left[w^{\downarrow}(t)\right]^{p}}{V_{*}(s)}, \sup _{s \geq t} \frac{\left[w^{\downarrow}(s)\right]^{p}}{V_{*}(s)}\right)=\sup _{s \geq t} \frac{\left[w^{\downarrow}(s)\right]^{p}}{V_{*}(s)} .
\end{aligned}
$$

Similarly, using the monotonicity of $V_{*}$, we find

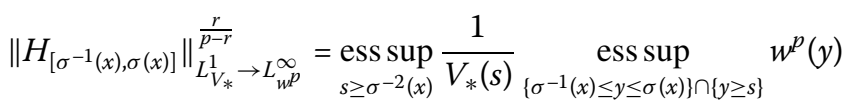

$$
\begin{aligned}
& =\sup _{\sigma^{-1}(x) \leq s \leq \sigma(x)} \frac{1}{V_{*}(s)} \sup _{\sigma^{-1}(x) \leq y \leq \sigma(x)} \underset{s \leq y \leq \sigma(x)}{\operatorname{ess} \sup } w^{p}(y) \\
& =\underset{\sigma^{-1}(x) \leq y \leq \sigma(x)}{\operatorname{ess} \sup } \frac{w^{p}(y)}{V_{*}(y)}
\end{aligned}
$$

and the estimate $\mathscr{A}_{1} \approx \mathscr{A}_{1}^{\prime}$ follows.

For the second part we observe that $C_{S} \approx \mathscr{B}_{0}^{\prime}+\mathscr{B}_{1}^{\prime}$, where $\mathscr{B}_{0}^{\prime}$ is the least constant in the inequality

$$
\left(\int_{0}^{\infty} u(x)\left[\operatorname{ess}_{x \leq y \leq \sigma^{2}(x)} w(y)\right]^{r}\left(\int_{\sigma^{2}(x)}^{\infty} h\right)^{\frac{r}{p}} d x\right)^{\frac{p}{r}} \leq\left[\mathscr{B}_{0}^{\prime}\right]^{p}\|h\|_{L_{V}^{1}}, \quad h \in \mathfrak{M}^{+},
$$

and

$$
\left[\mathscr{B}_{1}^{\prime}\right]^{p}= \begin{cases}\sup _{t>0}\left(\int_{0}^{t} u\right)^{\frac{p}{r}}\left\|H_{t}^{*}\right\|_{L_{V}^{1} \rightarrow L_{w p}^{\infty},}, & r \geq p, \\ \left(\int_{0}^{\infty} u(x)\left(\int_{0}^{x} u\right)^{\frac{r}{p-r}}\left\|H_{\left[\sigma^{-1}(x), \sigma(x)\right]}^{*}\right\|_{L_{V}^{\frac{r}{p-r}} \rightarrow L_{w^{p}}^{\infty}}^{\infty} d x\right)^{\frac{p-r}{r}}, & 0<r<p .\end{cases}
$$

By a change of variables we see that (4.7) is equivalent to

$$
\left(\int_{0}^{\infty} u(x)\left[\operatorname{ess}_{x \leq y \leq \sigma^{2}(x)} w(y)\right]^{r}\left(\int_{x}^{\infty} h\right)^{\frac{r}{p}} d x\right)^{\frac{p}{r}} \leq\left[\mathscr{B}_{0}^{\prime}\right]^{p}\|h\|_{L_{V_{\sigma^{2}}}^{1}}, \quad h \in \mathfrak{M}^{+},
$$

where $V_{\sigma^{2}}(y):=V\left(\sigma^{2}(t)\right)$. By the same argument as above it follows that $\mathscr{B}_{0}^{\prime} \approx \mathscr{B}_{0}$ and $\mathscr{B}_{1}^{\prime} \approx \mathscr{B}_{1}$.

Analogously, we obtain the sharp estimates for the best constants in (1.4) and (1.5).

Suppose for simplicity that $0<\int_{t}^{\infty} u<\infty$ for all $t>0, \int_{0}^{\infty} u=\infty$ and define the functions $\zeta:[0 ; \infty) \rightarrow[0 ; \infty), \zeta^{-1}:[0 ; \infty) \rightarrow[0 ; \infty)$, by

$$
\begin{aligned}
& \zeta(x):=\sup \left\{y>0: \int_{y}^{\infty} u \geq \frac{1}{2} \int_{x}^{\infty} u\right\}, \\
& \zeta^{-1}(x):=\sup \left\{y>0: \int_{y}^{\infty} u \geq 2 \int_{x}^{\infty} u\right\} .
\end{aligned}
$$


Let $\zeta^{2}:=\zeta(\zeta)$. For $0 \leq c<d<\infty$ and $h \in \mathfrak{M}^{+}$we put

$$
\begin{aligned}
& \mathscr{H}_{d} h(x):=\chi_{(0, d]}(x) \int_{x}^{\infty} h, \\
& \mathscr{H}_{c, d} h(x):=\chi_{(c, d]}(x) \int_{x}^{\zeta(d)} h, \\
& \mathscr{H}_{c}^{*} h(x):=\chi_{(0, d]}(x) \int_{0}^{x} h, \\
& \mathscr{H}_{c, d}^{*} h(x):=\chi_{(c, d]}(x) \int_{\zeta^{-1}(c)}^{x} h .
\end{aligned}
$$

We need the following partial cases of [21], Theorems 3.1 and 3.2.

Theorem 4.3 Let $0<r<\infty$. Then:

(a) For validity of the inequality

$$
\left(\int_{0}^{\infty}\left[\underset{0 \leq y \leq x}{\operatorname{ess} \sup } w_{0}(y) \int_{y}^{\infty} h\right]^{r} u(x) d x\right)^{\frac{1}{r}} \leq C_{2}\|h\|_{L_{v_{0}}^{1}}, \quad h \in \mathfrak{M}^{+},
$$

it is necessary and sufficient that the inequality

$$
\left(\int_{0}^{\infty} u(x)\left[w_{0}^{\uparrow}(x)\right]^{r}\left(\int_{x}^{\infty} h\right)^{r} d x\right)^{\frac{1}{r}} \leq D_{0}\|h\|_{L_{\nu_{0}}^{1}}, \quad h \in \mathfrak{M}^{+},
$$

holds and the constant

$$
D_{1}:= \begin{cases}\sup _{t>0}\left(\int_{t}^{\infty} u\right)^{\frac{1}{r}}\left\|\mathscr{H}_{t}\right\|_{L_{v_{0}}^{1} \rightarrow L_{w_{0}}^{\infty},} & r \geq 1, \\ \left(\int_{0}^{\infty} u(x)\left(\int_{x}^{\infty} u\right)^{\frac{r}{1-r}}\left\|\mathscr{H}_{\left[\zeta^{-1}(x), \zeta(x)\right]}\right\|_{L_{v_{0}}^{1} \rightarrow L_{w_{0}}^{\infty}}^{\frac{r}{1-r}} d x\right)^{\frac{1-r}{r}}, & 0<r<1,\end{cases}
$$

is finite. Moreover, $C_{2} \approx D_{0}+D_{1}$.

(b) For validity of the inequality

$$
\left(\int_{0}^{\infty}\left[\underset{0 \leq y \leq x}{\operatorname{ess} \sup } w_{0}(y) \int_{0}^{y} h\right]^{r} u(x) d x\right)^{\frac{1}{r}} \leq C_{3}\|h\|_{L_{\nu_{0}}^{1}}, \quad h \in \mathfrak{M}^{+},
$$

it is necessary and sufficient that the inequality

$$
\left(\int_{0}^{\infty} u(x)\left[\operatorname{esssp}_{\zeta^{-2}(x) \leq y \leq x} w_{0}(y)\right]^{r}\left(\int_{0}^{\zeta^{-2}(x)} h\right)^{r} d x\right)^{\frac{1}{r}} \leq E_{0}\|h\|_{L_{\nu_{0}}^{1}}, \quad h \in \mathfrak{M}^{+},
$$

holds and the constant

$$
E_{1}:= \begin{cases}\sup _{t>0}\left(\int_{t}^{\infty} u\right)^{\frac{1}{r}}\left\|\mathscr{H}_{t}^{*}\right\|_{L_{\nu_{0}}^{1} \rightarrow L_{w_{0}}^{\infty},} & r \geq 1, \\ \left(\int_{0}^{\infty} u(x)\left(\int_{x}^{\infty} u\right)^{\frac{r}{1-r}}\left\|\mathscr{H}_{\left[\zeta^{-1}(x), \zeta(x)\right]}^{*}\right\|_{L_{v_{0}}^{1} \rightarrow L_{w_{0}}^{\infty}}^{\frac{r}{1-r}} d x\right)^{\frac{1-r}{r}}, & 0<r<1,\end{cases}
$$

is finite. Moreover, $C_{3} \approx E_{0}+E_{1}$. 
Using Theorem 4.3 we characterize (1.4) and (1.5) with $k(x, y)=1$.

Theorem 4.4 Let $0<p, r<\infty$ and $k(x, y)=1$. Then for the best possible constants of the inequalities (1.4) and (1.5) the following equivalences hold:

$$
C_{\mathscr{T}} \approx \mathscr{D}_{0}+\mathscr{D}_{1}, \quad C_{\mathscr{S}} \approx \mathscr{E}_{0}+\mathscr{E}_{1}
$$

where

$$
\begin{aligned}
& \mathscr{D}_{0}=\sup _{t>0}[V(t)]^{-\frac{1}{p}}\left(\int_{0}^{t} u\left[w^{\uparrow}\right]^{r}\right)^{\frac{1}{r}}, \quad r \geq p, \\
& \mathscr{D}_{0}=\left(\int_{0}^{\infty}\left([V(x)]^{-1} \int_{0}^{x} u\left[w^{\uparrow}\right]^{r}\right)^{\frac{r}{p-r}} u(x)\left[w^{\uparrow}(x)\right]^{r} d x\right)^{\frac{p-r}{p r}}, \quad 0<r<p, \\
& \mathscr{D}_{1}=\sup _{t>0}\left(\int_{t}^{\infty} u\right)^{\frac{1}{r}} \sup _{0<y<t} \frac{w^{\uparrow}(y)}{[V(y)]^{\frac{1}{p}}}, \quad r \geq p \text {, } \\
& \mathscr{D}_{1}=\left(\int_{0}^{\infty} u(x)\left(\int_{x}^{\infty} u\right)^{\frac{r}{p-r}}\left(\operatorname{ess~sup}_{\zeta^{-1}(x) \leq y \leq \zeta(x)} \frac{[w(y)]^{p}}{V(y)}\right)^{\frac{r}{p-r}} d x\right)^{\frac{p-r}{p r}}, \quad 0<r<p, \\
& \mathscr{E}_{0}=\sup _{t>0}\left[V_{*}\left(\zeta^{-2}(t)\right)\right]^{-\frac{1}{p}}\left(\int_{t}^{\infty} u(x)\left[\operatorname{esssup}_{\zeta^{-2}(x) \leq y \leq x} w(y)\right]^{r} d x\right)^{\frac{1}{r}}, \quad r \geq p, \\
& \mathscr{E}_{0}=\left(\int_{0}^{\infty}\left(\left[V_{*}\left(\zeta^{-2}(z)\right)\right]^{-1} \int_{z}^{\infty} u(x)\left[\operatorname{ess~sup}_{\zeta^{-2}(x) \leq y \leq x} w(y)\right]^{r} d x\right)^{\frac{r}{p-r}}\right. \\
& \left.\times u(z)\left[\operatorname{ess~sup}_{\zeta^{-2}(z) \leq y \leq z} w(y)\right]^{r} d z\right)^{\frac{p-r}{p r}}, \quad 0<r<p, \\
& \mathscr{E}_{1}=\sup _{t>0}\left(\int_{t}^{\infty} u\right)^{\frac{1}{r}} \underset{0 \leq y \leq t}{\operatorname{ess} \sup } \frac{w(y)}{\left[V_{*}(y)\right]^{\frac{1}{p}}}, \quad r \geq p, \\
& \mathscr{E}_{1}=\left(\int_{0}^{\infty} u(x)\left(\int_{x}^{\infty} u\right)^{\frac{r}{p-r}}\left(\operatorname{essup}_{\zeta^{-1}(x) \leq y \leq \zeta(x)} \frac{[w(y)]^{p}}{V_{*}(y)}\right)^{\frac{r}{p-r}} d x\right)^{\frac{p-r}{p r}}, \quad 0<r<p .
\end{aligned}
$$

\section{Main results}

To deal with the kernel transformation we need the following extension of Theorem 4.1 following from [21], Theorems 4.1 and 4.3 .

Theorem 5.1 Let $0<r<\infty, u, v_{0}, w_{0} \in \mathfrak{M}^{+}$and $k_{0}(x, y)$ satisfies Oinarov's condition (1.1). Then:

(a) For validity of the inequality

$$
\left(\int_{0}^{\infty}\left[\underset{y \geq x}{\operatorname{ess} \sup } k_{0}(y, x) w_{0}(y) \int_{0}^{y} h\right]^{r} u(x) d x\right)^{\frac{1}{r}} \leq \mathbf{C}_{0}\|h\|_{L_{v_{0}}^{1}}, \quad h \in \mathfrak{M}^{+},
$$

it is necessary and sufficient that the inequalities

$$
\left(\int_{0}^{\infty} u(x)\left[\underset{y \geq x}{\operatorname{ess} \sup } k_{0}(y, x) w_{0}(y)\right]^{r}\left(\int_{0}^{x} h\right)^{r} d x\right)^{\frac{1}{r}} \leq \mathbf{A}_{0}\|h\|_{L_{v_{0}}^{1}}, \quad h \in \mathfrak{M}^{+},
$$


and

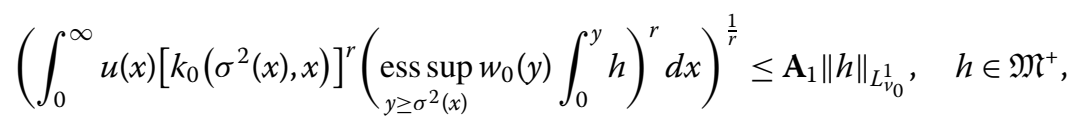

hold and the constant

$$
\mathbf{A}_{2}:= \begin{cases}\sup _{t>0}\left(\int_{0}^{t} u\right)^{\frac{1}{r}}\left\|H_{t}\right\|_{L_{v_{0}}^{1} \rightarrow L_{w_{0}(\cdot) k_{0}(\cdot, t)}^{\infty}, \frac{r}{1-r}} & r \geq 1, \\ \left(\int_{0}^{\infty} u(x)\left(\int_{0}^{x} u\right)^{\frac{r}{1-r}}\left\|H_{\left[\sigma^{-1}(x), \sigma^{2}(x)\right]}\right\|_{L_{v_{0}}^{1} \rightarrow L_{w_{0}(\cdot) k_{0}\left(\cdot, \sigma^{-1}(x)\right)}^{\infty}}^{1} d x\right)^{\frac{1-r}{r}}, & r<1,\end{cases}
$$

is finite. Moreover, $\mathbf{C}_{0} \approx \mathbf{A}_{0}+\mathbf{A}_{1}+\mathbf{A}_{2}$.

(b) For validity of the inequality

$$
\left(\int_{0}^{\infty}\left[\underset{y \geq x}{\operatorname{ess} \sup } k_{0}(y, x) w_{0}(y) \int_{y}^{\infty} h\right]^{r} u(x) d x\right)^{\frac{1}{r}} \leq \mathbf{C}_{1}\|h\|_{L_{v_{0}}^{1}}, \quad h \in \mathfrak{M}^{+}
$$

it is necessary and sufficient that the inequalities

$$
\left(\int_{0}^{\infty} u(x)\left[\operatorname{ess~sup~}_{x \leq y \leq \sigma^{3}(x)} k_{0}(y, x) w_{0}(y)\right]^{r}\left(\int_{\sigma^{3}(x)}^{\infty} h\right)^{r} d x\right)^{\frac{1}{r}} \leq \mathbf{B}_{0}\|h\|_{L_{v_{0}}^{1}}, \quad h \in \mathfrak{M}^{+}
$$

and

$$
\left(\int_{0}^{\infty} u(x)\left[k_{0}\left(\sigma^{2}(x), x\right)\right]^{r}\left(\operatorname{esssup}_{y \geq \sigma^{2}(x)} w_{0}(y) \int_{y}^{\infty} h\right)^{r} d x\right)^{\frac{1}{r}} \leq \mathbf{B}_{1}\|h\|_{L_{v_{0}}^{1}}, \quad h \in \mathfrak{M}^{+},
$$

hold and the constant

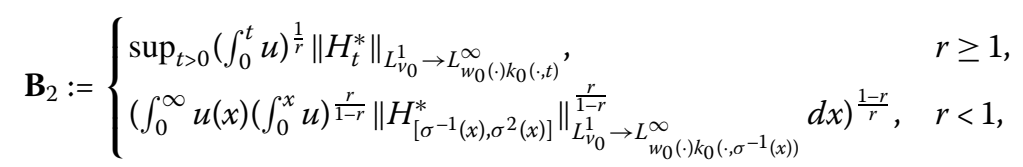

is finite. Moreover, $\mathbf{C}_{0} \approx \mathbf{B}_{0}+\mathbf{B}_{1}+\mathbf{B}_{2}$.

Using Theorem 5.1 we obtain the characterization of (1.2) and (1.3) for $0<p, r<\infty$. Denote

$$
\begin{aligned}
& W_{k}(x):=\underset{y \geq x}{\operatorname{ess} \sup } k(y, x) w(y), \quad \mathscr{W}_{k}(x):=\underset{x \leq y \leq \sigma^{3}(x)}{\operatorname{ess} \sup } k(y, x) w(y), \\
& \mathbf{w}_{\sigma}(w)(x):=\underset{x \leq y \leq \sigma^{2}(x)}{\operatorname{ess} \sup } w(y), \\
& k_{\sigma}(x):=k\left(\sigma^{2}(x), x\right), \quad g_{\sigma^{k}}(y):=g\left(\sigma^{k}(y)\right), \\
& \sigma_{0}(x):=\inf \left\{y>0: \int_{0}^{y} u\left[k_{\sigma}\right]^{r} \geq 2 \int_{0}^{x} u\left[k_{\sigma}\right]^{r}\right\} \\
& \sigma_{0}^{-1}(x):=\inf \left\{y>0: \int_{0}^{y} u\left[k_{\sigma}\right]^{r} \geq \frac{1}{2} \int_{0}^{x} u\left[k_{\sigma}\right]^{r}\right\} .
\end{aligned}
$$


Person et al. Journal of Inequalities and Applications (2016) 2016:237

Page 13 of 18

Theorem 5.2 Let $0<p, r<\infty$. Then, for the best possible constants of the inequalities (1.2) and (1.3) the following equivalences hold:

$$
C_{T} \approx \mathbb{A}_{0}+\mathbb{A}_{1,0}+\mathbb{A}_{1,1}+\mathbb{A}_{2}, \quad C_{S} \approx \mathbb{B}_{0}+\mathbb{B}_{1,0}+\mathbb{B}_{1,1}+\mathbb{B}_{2}
$$

where

$$
\begin{aligned}
& \mathbb{A}_{0}=\sup _{t>0}\left[V_{*}(t)\right]^{-\frac{1}{p}}\left(\int_{t}^{\infty} u\left[W_{k}\right]^{r}\right)^{\frac{1}{r}}, \quad r \geq p, \\
& \mathbb{A}_{0}=\left(\int_{0}^{\infty}\left(\left[V_{*}(x)\right]^{-1} \int_{x}^{\infty} u\left[W_{k}\right]^{r}\right)^{\frac{r}{p-r}} u(x)\left[W_{k}(x)\right]^{r} d x\right)^{\frac{p-r}{p r}}, \quad 0<r<p, \\
& \mathbb{A}_{1,0}=\sup _{t>0}\left[\left(V_{*}\right)_{\sigma^{2}}(t)\right]^{-\frac{1}{p}}\left(\int_{t}^{\infty} u\left[k_{\sigma} w_{\sigma^{2}}^{\downarrow}\right]^{r}\right)^{\frac{1}{r}}, \quad r \geq p, \\
& \mathbb{A}_{1,0}=\left(\int_{0}^{\infty}\left(\left[\left(V_{*}\right)_{\sigma^{2}}(x)\right]^{-1} \int_{x}^{\infty} u\left[k_{\sigma} w_{\sigma^{2}}^{\downarrow}\right]^{r}\right)^{\frac{r}{p-r}}\right. \\
& \left.\times u(x)\left[k_{\sigma}(x) w_{\sigma^{2}}^{\downarrow}(x)\right]^{r} d x\right)^{\frac{p-r}{p r}}, \quad 0<r<p, \\
& \mathbb{A}_{1,1}=\sup _{t>0}\left(\int_{0}^{t} u\left[k_{\sigma}\right]^{r}\right)^{\frac{1}{r}} \operatorname{ess} \sup \frac{w_{\sigma^{2}}(y)}{\left[\left(V_{*}\right)_{\sigma^{2}}(y)\right]^{\frac{1}{p}}}, \quad r \geq p, \\
& \mathbb{A}_{1,1}=\left(\int_{0}^{\infty} u(x)\left[k_{\sigma}(x)\right]^{r}\left(\int_{0}^{x} u\left[k_{\sigma}\right]^{r}\right)^{\frac{r}{p-r}}\right. \\
& \left.\times\left(\operatorname{ess~sup}_{\sigma_{0}^{-1}(x) \leq y \leq \sigma_{0}(x)} \frac{\left[w_{\sigma^{2}}(y)\right]^{p}}{\left(V_{*}\right)_{\sigma^{2}}(y)}\right)^{\frac{r}{p-r}} d x\right)^{\frac{p-r}{p r}}, \quad 0<r<p, \\
& \mathbb{A}_{2}=\sup _{t>0}\left(\int_{0}^{t} u\right)^{\frac{1}{r}} \operatorname{ess\operatorname {sup}} \frac{w(y) k(y, t)}{\left[V_{*}(y)\right]^{\frac{1}{p}}}, \quad r \geq p, \\
& \mathbb{A}_{2}=\left(\int_{0}^{\infty} u(x)\left(\int_{0}^{x} u\right)^{\frac{r}{p-r}}\right. \\
& \left.\times\left(\operatorname{ess}_{\sigma^{-1}(x) \leq y \leq \sigma^{2}(x)} \frac{\left[w(y) k\left(y, \sigma^{-1}(x)\right)\right]^{p}}{V_{*}(y)}\right)^{\frac{r}{p-r}} d x\right)^{\frac{p-r}{p r}}, \quad 0<r<p, \\
& \mathbb{B}_{0}=\sup _{t>0}\left[V_{\sigma^{3}}(t)\right]^{-\frac{1}{p}}\left(\int_{0}^{t} u\left[\mathscr{W}_{k}\right]^{r}\right)^{\frac{1}{r}}, \quad r \geq p, \\
& \mathbb{B}_{0}=\left(\int_{0}^{\infty}\left(\left[V_{\sigma^{3}}(z)\right]^{-1} \int_{0}^{z} u\left[\mathscr{W}_{k}\right]^{r}\right)^{\frac{r}{p-r}} u(z)\left[\mathscr{W}_{k}(z)\right]^{r} d z\right)^{\frac{p-r}{p r}}, \quad 0<r<p, \\
& \mathbb{B}_{1,0}=\sup _{t>0}\left[V_{\sigma^{3}}\left(\sigma_{0}^{2}(t)\right)\right]^{-\frac{1}{p}}\left(\int_{0}^{t} u\left[k_{\sigma} \mathbf{w}_{\sigma_{0}}\left(w_{\sigma^{3}}\right)\right]^{r}\right)^{\frac{1}{r}}, \quad r \geq p, \\
& \mathbb{B}_{1,0}=\left(\int_{0}^{\infty}\left(\left[V_{\sigma^{2}}\left(\sigma_{0}^{2}(t)\right)(z)\right]^{-1} \int_{0}^{z} u\left[k_{\sigma}\right]^{r}\left[\mathbf{w}_{\sigma_{0}}\left(w_{\sigma^{3}}\right)\right]^{r}\right)^{\frac{r}{p-r}}\right. \\
& \left.\times u(z)\left[k_{\sigma}(z) \mathbf{w}_{\sigma_{0}}\left(w_{\sigma^{3}}\right)(z)\right]^{r} d z\right)^{\frac{p-r}{p r}}, \quad 0<r<p,
\end{aligned}
$$




$$
\begin{aligned}
\mathbb{B}_{1,1}= & \sup _{t>0}\left(\int_{0}^{t} u\left[k_{\sigma}\right]^{r}\right)^{\frac{1}{r}} \operatorname{esssup} \frac{w_{\sigma^{3}}(y)}{\left[V_{\sigma^{3}}(y)\right]^{\frac{1}{p}}}, \quad r \geq p, \\
\mathbb{B}_{1,1}= & \left(\int_{0}^{\infty} u(x)\left[k_{\sigma}(x)\right]^{r}\left(\int_{0}^{x} u\left[k_{\sigma}\right]^{r}\right)^{\frac{r}{p-r}}\right. \\
& \left.\times\left(\operatorname{esssup}_{\sigma_{0}^{-1}(x) \leq y \leq \sigma_{0}(x)} \frac{\left[w_{\sigma^{3}}(y)\right]^{p}}{V_{\sigma^{3}}(y)}\right)^{\frac{r}{p-r}} d x\right)^{\frac{p-r}{p r}}, \quad 0<r<p, \\
\mathbb{B}_{2}= & \sup _{t>0}\left(\int_{0}^{t} u\right)^{\frac{1}{r}} \operatorname{ess} \sup \frac{w(y) k(y, t)}{[V(y)]^{\frac{1}{p}}}, \quad r \geq p, \\
\mathbb{B}_{2}= & \left(\int_{0}^{\infty} u(x)\left(\int_{0}^{x} u\right)^{\frac{r}{p-r}}\right. \\
& \left.\times\left(\operatorname{ess}_{\sigma^{-1}(x) \leq y \leq \sigma^{2}(x)} \frac{\left[w(y) k\left(y, \sigma^{-1}(x)\right)\right]^{p}}{V(y)}\right)^{\frac{r}{p-r}} d x\right)^{\frac{p-r}{p r}}, \quad 0<r<p .
\end{aligned}
$$

Proof We start with the inequality (1.2). Since (1.2) $\Leftrightarrow$ (2.1), then applying Theorem 5.1 we see that

$$
C_{T} \approx \mathbb{A}_{0}^{\prime}+\mathbb{A}_{1}^{\prime}+\mathbb{A}_{2}^{\prime}
$$

where $\mathbb{A}_{0}^{\prime}$ and $\mathbb{A}_{1}^{\prime}$ are the best constants in the inequalities

$$
\begin{aligned}
& \left(\int_{0}^{\infty} u(x)[\underset{y \geq x}{\operatorname{ess} \sup } k(y, x) w(y)]^{r}\left(\int_{0}^{x} h\right)^{\frac{r}{p}} d x\right)^{\frac{p}{r}} \leq\left[\mathbb{A}_{0}^{\prime}\right]^{p}\|h\|_{L_{V_{*}}^{1}}, \quad h \in \mathfrak{M}^{+}, \\
& \left(\int_{0}^{\infty} u(x)\left[k\left(\sigma^{2}(x), x\right)\right]^{r}\left(\underset{y \geq \sigma^{2}(x)}{\operatorname{ess} \sup }[w(y)]^{p} \int_{0}^{y} h\right)^{\frac{r}{p}} d x\right)^{\frac{p}{r}} \leq\left[\mathbb{A}_{1}^{\prime}\right]^{p}\|h\|_{L_{V_{*}}^{1}}, \quad h \in \mathfrak{M}^{+},
\end{aligned}
$$

and

$$
\left[\mathbb{A}_{2}^{\prime}\right]^{p}:= \begin{cases}\sup _{t>0}\left(\int_{0}^{t} u\right)^{\frac{p}{r}}\left\|H_{t}\right\|_{L_{V_{*}}^{1} \rightarrow L_{[w(\cdot) k(\cdot, t)]^{p}}^{\infty},} \|^{\frac{r}{p-r}} & r \geq p, \\ \left(\int_{0}^{\infty} u(x)\left(\int_{0}^{x} u\right)^{\frac{r}{p-r}} \|\left. H_{\left[\sigma^{-1}(x), \sigma^{2}(x)\right]}\right|_{\left.L_{V_{*}}^{1} \rightarrow L_{\left[w(\cdot) k\left(\cdot, \sigma^{-1}(x)\right)\right]^{p}}^{\infty} d x\right)^{\frac{p-r}{r}},}, 0<r<p .\right.\end{cases}
$$

Applying (4.5) and (4.6) we see that $\mathbb{A}_{0}^{\prime} \approx \mathbb{A}_{0}$ and $\mathbb{A}_{2}^{\prime} \approx \mathbb{A}_{2}$. By a change of variable we find that (5.4) is equivalent to the inequality

$$
\begin{aligned}
& \left(\int_{0}^{\infty} u(x)\left[k_{\sigma}(x)\right]^{r}\left(\underset{y \geq x}{\operatorname{ess} \sup }\left[w_{\sigma^{2}}(y)\right]^{p} \int_{0}^{y} h\right)^{\frac{r}{p}} d x\right)^{\frac{p}{r}} \\
& \quad \leq\left[\mathbb{A}_{1}^{\prime}\right]^{p}\|h\|_{L_{\left[V_{*}\right]^{2}}^{1}}, \quad h \in \mathfrak{M}^{+},
\end{aligned}
$$

which is governed by Theorem 4.1. Arguing analogously to the proof of Theorem 4.2 we see that

$$
\mathbb{A}_{1}^{\prime} \approx \mathbb{A}_{1,0}^{\prime}+\mathbb{A}_{1,1}^{\prime},
$$


where $\mathbb{A}_{1,0}^{\prime}$ is the best constant of the inequality

$$
\left(\int_{0}^{\infty} u(x)\left[k_{\sigma}(x)\right]^{r}\left[w_{\sigma^{2}}^{\downarrow}(x)\right]^{r}\left(\int_{0}^{x} h\right)^{\frac{r}{p}} d x\right)^{\frac{p}{r}} \leq\left[\mathbb{A}_{1,0}^{\prime}\right]^{p}\|h\|_{L_{\left[V_{*}\right]^{2}}^{1}}, \quad h \in \mathfrak{M}^{+},
$$

and

$$
\begin{aligned}
& {\left[\mathbb{A}_{1,1}^{\prime}\right]^{p}:=\sup _{t>0}\left(\int_{0}^{t} u\left[k_{\sigma}\right]^{r}\right)^{\frac{p}{r}}\left\|H_{t}\right\|_{L_{\left[V_{*}\right]_{\sigma}}^{1} \rightarrow L_{w_{\sigma^{2}}}^{\infty}, \quad r \geq p,},} \\
& {\left[\mathbb{A}_{1,1}^{\prime}\right]^{p}:=\left(\int_{0}^{\infty} u(x)\left[k_{\sigma}(x)\right]^{r}\left(\int_{0}^{x} u\left[k_{\sigma}\right]^{r}\right)^{\frac{r}{p-r}}\left\|H_{\left[\sigma_{0}^{-1}(x), \sigma_{0}(x)\right]}\right\|_{L_{\left[V_{*}\right]^{2}}^{\frac{r}{p-r}} \rightarrow L_{\sigma_{\sigma^{p}}}^{\infty}} d x\right)^{\frac{p-r}{r}},}
\end{aligned}
$$

for $0<r<p$. Again applying (4.5) and (4.6) we see that $\mathbb{A}_{1,0}^{\prime} \approx \mathbb{A}_{1,0}$ and $\mathbb{A}_{1,1}^{\prime} \approx \mathbb{A}_{1,1}$.

The proof for the inequality (1.3) is similar.

Analogously, we obtain the sharp estimates for the best constants in (1.4) and (1.5). To this end we need the following extension of Theorem 4.3 from [21], Theorems 5.1 and 5.2.

Theorem 5.3 Let $0<r<\infty, u, v_{0}, w_{0} \in \mathfrak{M}^{+}$and $k_{0}(x, y)$ satisfy Oinarov's condition (1.1).

Then:

(a) For validity of the inequality

$$
\left(\int_{0}^{\infty}\left[\underset{0 \leq y \leq x}{\operatorname{ess} \sup } k_{0}(x, y) w_{0}(y) \int_{y}^{\infty} h\right]^{r} u(x) d x\right)^{\frac{1}{r}} \leq \mathbf{C}_{0}\|h\|_{L_{\nu_{0}}^{1}}, \quad h \in \mathfrak{M}^{+}
$$

it is necessary and sufficient that the inequalities

$$
\left(\int_{0}^{\infty} u(x)\left[\operatorname{ess}_{0 \leq y \leq x} k_{0}(x, y) w_{0}(y)\right]^{r}\left(\int_{x}^{\infty} h\right)^{r} d x\right)^{\frac{1}{r}} \leq \mathbf{A}_{0}\|h\|_{L_{v_{0}}^{1}}, \quad h \in \mathfrak{M}^{+},
$$

and

$$
\begin{aligned}
& \left(\int_{0}^{\infty} u(x)\left[k_{0}\left(x, \zeta^{-2}(x)\right)\right]^{r}\left(\operatorname{essup}_{0 \leq y \leq \zeta^{-2}(x)} w_{0}(y) \int_{y}^{\infty} h\right)^{r} d x\right)^{\frac{1}{r}} \\
& \quad \leq \mathbf{A}_{1}\|h\|_{L_{v_{0}}^{1}}, \quad h \in \mathfrak{M}^{+},
\end{aligned}
$$

hold and the constant

$$
\mathbf{A}_{2}:= \begin{cases}\sup _{t>0}\left(\int_{t}^{\infty} u\right)^{\frac{1}{r}}\left\|\mathscr{H}_{t}\right\|_{L_{\nu_{0}}^{1} \rightarrow L_{w_{0}(\cdot) k_{0}(t,)^{\prime}}^{\infty}} \|^{\frac{r}{1-r}} & r \geq 1, \\ \left(\int_{0}^{\infty} u(x)\left(\int_{x}^{\infty} u\right)^{\frac{r}{1-r}}\left\|\mathscr{H}_{\left[\zeta^{-1}(x), \zeta^{2}(x)\right]}\right\|_{\left.L_{\nu_{0}}^{1} \rightarrow L_{w_{0}(\cdot) k_{0}\left(\zeta^{2}(x) \cdot\right)}^{\infty} d x\right)^{\frac{1-r}{r}},}, r<1,\right.\end{cases}
$$

is finite. Moreover, $\mathbf{C}_{0} \approx \mathbf{A}_{0}+\mathbf{A}_{1}+\mathbf{A}_{2}$.

(b) For validity of the inequality

$$
\left(\int_{0}^{\infty}\left[\underset{0 \leq y \leq x}{\operatorname{ess} \sup } k_{0}(y, x) w_{0}(y) \int_{0}^{y} h\right]^{r} u(x) d x\right)^{\frac{1}{r}} \leq \mathbf{C}_{1}\|h\|_{L_{v_{0}}^{1}}, \quad h \in \mathfrak{M}^{+},
$$


it is necessary and sufficient that the inequalities

$$
\left(\int_{0}^{\infty} u(x)\left[\operatorname{esssup}_{\zeta^{-3}(x) \leq y \leq x} k_{0}(x, y) w_{0}(y)\right]^{r}\left(\int_{0}^{\zeta^{-3}(x)} h\right)^{r} d x\right)^{\frac{1}{r}} \leq \mathbf{B}_{0}\|h\|_{L_{\nu_{0}}^{1}}, \quad h \in \mathfrak{M}^{+},
$$

and

$$
\left(\int_{0}^{\infty} u(x)\left[k_{0}\left(x, \zeta^{-2}(x)\right)\right]^{r}\left(\operatorname{ess~sup}_{0 \leq y \leq \zeta^{-2}(x)} w_{0}(y) \int_{0}^{y} h\right)^{r} d x\right)^{\frac{1}{r}} \leq \mathbf{B}_{1}\|h\|_{L_{\nu_{0}}^{1}}, \quad h \in \mathfrak{M}^{+},
$$

hold and the constant

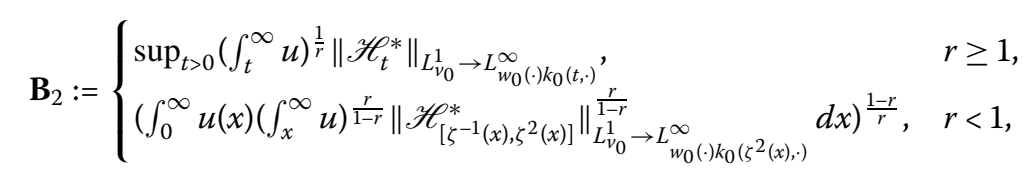

is finite. Moreover, $\mathbf{C}_{0} \approx \mathbf{B}_{0}+\mathbf{B}_{1}+\mathbf{B}_{2}$.

Using Theorem 5.3 we obtain the characterization of (1.4) and (1.5) for $0<p, r<\infty$. Denote

$$
\begin{aligned}
& W_{k}^{*}(x):=\underset{0 \leq y \leq x}{\operatorname{ess} \sup } k(x, y) w(y), \quad \mathscr{W}_{k}^{*}(x):=\underset{\zeta^{-3}(x) \leq y \leq x}{\operatorname{ess} \sup } k(x, y) w(y) \\
& \Omega_{\zeta}(w)(x):=\underset{\zeta^{-2}(x) \leq y \leq x}{\operatorname{ess} \sup } w(y) \\
& k_{\zeta}(x):=k\left(x, \zeta^{-2}(x)\right), \quad g_{\zeta^{-k}}(y):=g\left(\zeta^{-k}(y)\right) \\
& \zeta_{0}(x):=\sup \left\{y>0: \int_{y}^{\infty} u\left[k_{\zeta}\right]^{r} \geq \frac{1}{2} \int_{x}^{\infty} u\left[k_{\zeta}\right]^{r}\right\} \\
& \zeta_{0}^{-1}(x):=\sup \left\{y>0: \int_{y}^{\infty} u\left[k_{\zeta}\right]^{r} \geq 2 \int_{x}^{\infty} u\left[k_{\zeta}\right]^{r}\right\}
\end{aligned}
$$

Theorem 5.4 Let $0<p, r<\infty$. Then for the best possible constants of the inequalities (1.4) and (1.5) the following equivalences hold:

$$
C_{\mathscr{T}} \approx \mathbb{D}_{0}+\mathbb{D}_{1,0}+\mathbb{D}_{1,1}+\mathbb{D}_{2}, \quad C_{\mathscr{S}} \approx \mathbb{E}_{0}+\mathbb{E}_{1,0}+\mathbb{E}_{1,1}+\mathbb{E}_{2}
$$

where

$$
\begin{aligned}
& \mathbb{D}_{0}=\sup _{t>0}[V(t)]^{-\frac{1}{p}}\left(\int_{0}^{t} u\left[W_{k}^{*}\right]^{r}\right)^{\frac{1}{r}}, \quad r \geq p, \\
& \mathbb{D}_{0}=\left(\int_{0}^{\infty}\left([V(x)]^{-1} \int_{0}^{x} u\left[W_{k}^{*}\right]^{r}\right)^{\frac{r}{p-r}} u(x)\left[W_{k}^{*}(x)\right]^{r} d x\right)^{\frac{p-r}{p r}}, \quad 0<r<p, \\
& \mathbb{D}_{1,0}=\sup _{t>0}\left[V_{\zeta^{-2}}(t)\right]^{-\frac{1}{p}}\left(\int_{0}^{t} u\left[k_{\zeta} w_{\zeta^{-2}}^{\uparrow}\right]^{r}\right)^{\frac{1}{r}}, \quad r \geq p, \\
& \mathbb{D}_{1,0}=\left(\int_{0}^{\infty}\left(\left[V_{\zeta^{-2}}(x)\right]^{-1} \int_{0}^{x} u\left[k_{\zeta} w_{\zeta^{-2}}^{\uparrow}\right]^{r}\right)^{\frac{r}{p-r}}\right.
\end{aligned}
$$


Person et al. Journal of Inequalities and Applications (2016) 2016:237

Page 17 of 18

$$
\begin{aligned}
& \left.\times u(x)\left[k_{\zeta}(x) w_{\zeta^{-2}}^{\uparrow}(x)\right]^{r} d x\right)^{\frac{p-r}{p r}}, \quad 0<r<p, \\
& \mathbb{D}_{1,1}=\sup _{t>0}\left(\int_{t}^{\infty} u\left[k_{\zeta}\right]^{r}\right)^{\frac{1}{r}} \underset{0 \leq y \leq t}{\operatorname{ess} \sup } \frac{w_{\zeta^{-2}}(y)}{\left[V_{\zeta^{-2}}(y)\right]^{\frac{1}{p}}}, \quad r \geq p, \\
& \mathbb{D}_{1,1}=\left(\int_{0}^{\infty} u(x)\left[k_{\zeta}(x)\right]^{r}\left(\int_{x}^{\infty} u\left[k_{\zeta}\right]^{r}\right)^{\frac{r}{p-r}}\right. \\
& \left.\times\left(\operatorname{ess}_{\zeta_{0}^{-1}(x) \leq y \leq \zeta_{0}(x)} \frac{\left[w_{\zeta^{-2}}(y)\right]^{p}}{V_{\zeta^{-2}}(y)}\right)^{\frac{r}{p-r}} d x\right)^{\frac{p-r}{p r}}, \quad 0<r<p, \\
& \mathbb{D}_{2}=\sup _{t>0}\left(\int_{t}^{\infty} u\right)^{\frac{1}{r}} \underset{0 \leq y \leq t}{\operatorname{ess} \sup } \frac{w(y) k(t, y)}{[V(y)]^{\frac{1}{p}}}, \quad r \geq p, \\
& \mathbb{D}_{2}=\left(\int_{0}^{\infty} u(x)\left(\int_{x}^{\infty} u\right)^{\frac{r}{p-r}}\right. \\
& \left.\times\left(\operatorname{essip}_{\zeta^{-1}(x) \leq y \leq \zeta^{2}(x)} \frac{\left[w(y) k\left(\zeta^{2}(x), y\right)\right]^{p}}{V(y)}\right)^{\frac{r}{p-r}} d x\right)^{\frac{p-r}{p r}}, \quad 0<r<p, \\
& \mathbb{E}_{0}=\sup _{t>0}\left[\left(V_{*}\right)_{\zeta^{-3}}(t)\right]^{-\frac{1}{p}}\left(\int_{t}^{\infty} u\left[\mathscr{W}_{k}^{*}\right]^{r}\right)^{\frac{1}{r}}, \quad r \geq p, \\
& \mathbb{E}_{0}=\left(\int_{0}^{\infty}\left(\left[\left(V_{*}\right)_{\zeta^{-3}}(z)\right]^{-1} \int_{z}^{\infty} u\left[\mathscr{W}_{k}^{*}\right]^{r}\right)^{\frac{r}{p-r}} u(z)\left[\mathscr{W}_{k}^{*}(z)\right]^{r} d z\right)^{\frac{p-r}{p r}}, \quad 0<r<p, \\
& \mathbb{E}_{1,0}=\sup _{t>0}\left[\left(V_{*}\right)_{\zeta^{-2}}\left(\zeta_{0}^{-2}(t)\right)\right]^{-\frac{1}{p}}\left(\int_{t}^{\infty} u\left[k_{\zeta}\right]^{r}\left[\Omega_{\zeta_{0}}\left(w_{\zeta^{-2}}\right)\right]^{r}\right)^{\frac{1}{r}}, \quad r \geq p, \\
& \mathbb{E}_{1,0}=\left(\int_{0}^{\infty}\left(\left[\left(V_{*}\right)_{\zeta^{-2}}\left(\zeta_{0}^{-2}(t)\right)(z)\right]^{-1} \int_{z}^{\infty} u\left[k_{\zeta}\right]^{r}\left[\Omega_{\zeta_{0}}\left(w_{\zeta^{-2}}\right)\right]^{r}\right)^{\frac{r}{p-r}}\right. \\
& \left.\times u(z)\left[k_{\zeta}(z)\right]^{r}\left[\Omega_{\zeta_{0}}\left(w_{\zeta^{-2}}\right)\right]^{r} d z\right)^{\frac{p-r}{p r}}, \quad 0<r<p, \\
& \mathbb{E}_{1,1}=\sup _{t>0}\left(\int_{t}^{\infty} u\left[k_{\zeta}\right]^{r}\right)^{\frac{1}{r}} \underset{0 \leq y \leq t}{\operatorname{ess} \sup } \frac{w_{\zeta^{-2}}(y)}{\left[\left(V_{*}\right)_{\zeta^{-2}}(y)\right]^{\frac{1}{p}}}, \quad r \geq p, \\
& \mathbb{E}_{1,1}=\left(\int_{0}^{\infty} u(x)\left[k_{\zeta}(x)\right]^{r}\left(\int_{x}^{\infty} u\left[k_{\zeta}\right]^{r}\right)^{\frac{r}{p-r}}\right. \\
& \left.\times\left(\operatorname{ess}_{\zeta_{0}^{-1}(x) \leq y \leq \zeta_{0}(x)} \frac{\left[w_{\zeta^{-2}}(y)\right]^{p}}{\left(V_{*}\right)_{\zeta^{-2}}(y)}\right)^{\frac{r}{p-r}} d x\right)^{\frac{p-r}{p r}}, \quad 0<r<p, \\
& \mathbb{E}_{2}=\sup _{t>0}\left(\int_{t}^{\infty} u\right)^{\frac{1}{r}} \operatorname{ess} \sup \frac{w(y) k(t, y)}{\left[V_{*}(y)\right]^{\frac{1}{p}}}, \quad r \geq p, \\
& \mathbb{E}_{2}=\left(\int_{0}^{\infty} u(x)\left(\int_{x}^{\infty} u\right)^{\frac{r}{p-r}}\right. \\
& \left.\times\left(\operatorname{ess~sup}_{\zeta^{-1}(x) \leq y \leq \zeta^{2}(x)} \frac{\left[w(y) k\left(\zeta^{2}(x), y\right)\right]^{p}}{V_{*}(y)}\right)^{\frac{r}{p-r}} d x\right)^{\frac{p-r}{p r}}, \quad 0<r<p .
\end{aligned}
$$




\section{Competing interests}

The authors declare that they have no competing interests.

\section{Authors' contributions}

All the authors contributed equally and significantly in writing this paper. All the authors read and approved the final manuscript.

\section{Author details}

${ }^{1}$ Department of Engineering Sciences and Mathematics, Lulea University of Technology, Lulea, 97187, Sweden. ${ }^{2}$ UiT, The Arctic University of Norway, P.O. Box 385, Narvik, 8505, Norway. ${ }^{3}$ Department of Mathematics, Financial University under the Government of the Russian Federation, Leningradsky Prospekt 49, Moscow, 125993, Russia. ${ }^{4}$ Department of Nonlinear Analysis and Optimization, Peoples' Friendship University of Russia, Miklukho-Maklay 6, Moscow, 117198, Russia.

\section{Acknowledgements}

The research work of GE Shambilova and VD Stepanov was carried out at the Peoples' Friendship University of Russia and financially supported by the Russian Science Foundation (Project no. 16-41-02004).

\section{Received: 24 March 2016 Accepted: 7 September 2016 Published online: 28 September 2016}

\section{References}

1. Kufner, A, Persson, LE: Weighted Inequalities of Hardy Type. World Scientific, River Edge (2003)

2. Kufner, A, Maligranda, L, Persson, LE: The Hardy Inequality: About Its History and Some Related Results. Vydavatelsky Servis Publishing House, Pilsen (2007)

3. Kokalishvili, V, Meskhi, A, Persson, LE: Weighted Norm Inequalities for Integral Transforms with Product Kernels. Nova Science Publishers, New York (2010)

4. Kalybay, A, Person, LE, Temirkhanova, A: A new discrete Hardy-type inequality with kernels and monotone functions. J. Inequal. Appl. 2015, 321 (2015)

5. Machihara, S, Ozawa, T, Wadade, H: Scaling invariant Hardy inequalities of multiple logarithmic type on the whole space. J. Inequal. Appl. 2015, 281 (2015)

6. Mejjaoli, H: Hardy-type inequalities associated with the Weinstein operator. J. Inequal. Appl. 2015, 267 (2015)

7. Persson, L-E, Shambilova, GE, Stepanov, VD: Hardy-type inequalities on the weighted cones of quasi-concave functions. Banach J. Math. Anal. 9(2), 21-34 (2015)

8. Oguntuase, J, Fabelurin, O, Adeagbu-Sheikh, A, Persson, LE: Time scale Hardy inequalities with 'broken' exponent $p$. J. Inequal. Appl. 2015, 17 (2015)

9. Persson, LE, Shaimardan, S: Some new Hardy-type inequalities for Riemann-Liouville fractional q-integral operator J. Inequal. Appl. 2015, 296 (2015)

10. Gogatishvili, A, Opic, B, Pick, L: Weighted inequalities for Hardy-type operators involving suprema. Collect. Math. 57, 227-255 (2006)

11. Stepanov, VD: On a supremum operator. In: Spectral Theory, Function Spaces and Inequalities: New Techniques and Recent Trends. Operator Theory: Advances and Applications, vol. 219, pp. 233-242 (2012)

12. Gogatishvili, A, Pick, L: A reduction theorem for supremum operators. J. Comput. Appl. Math. 208, $270-279$ (2007)

13. Cwikel, M, Pustylnik, E: Weak type interpolation near 'endpoint' spaces. J. Funct. Anal. 171, 235-277 (2000)

14. Evans, WD, Opic, B: Real interpolation with logarithmic functions and reiteration. Can. J. Math. 52, 920-960 (2000)

15. Pick, L: Optimal Sobolev embeddings. Rudolph-Lipshitz-Vorlesungsreihe, no. 43. Rheinische Friedrich-Wilhelms-Universität Bonn (2002)

16. Prokhorov, DV: Inequalities for Riemann-Liouville operator involving suprema. Collect. Math. 61, 263-276 (2010)

17. Prokhorov, DV: Lorentz norm inequalities for the Hardy operator involving suprema. Proc. Am. Math. Soc. 140, 1585-1592 (2012)

18. Prokhorov, DV: Boundedness and compactness of a supremum-involving integral operator. Proc. Steklov Inst. Math. 283, 136-148 (2013)

19. Prokhorov, DV, Stepanov, VD: On weighted Hardy inequalities in mixed norms. Proc. Steklov Inst. Math. 283, 149-164 (2013)

20. Prokhorov, DV, Stepanov, VD: Estimates for a class of sublinear integral operators. Dokl. Math. 89, $372-377$ (2014)

21. Prokhorov, DV, Stepanov, VD: Weighted inequalities for quasilinear integral operators on the semiaxis and application to the Lorentz spaces. Sb. Math. 207(8), 135-162 (2016). doi:10.1070/SM8535

22. Krepela, M: Integral conditions for Hardy type operators involving suprema. Collect. Math. (2016). doi:10.1007/s13348-016-0170-6

23. Sinnamon, G: Transferring monotonicity in weighted norm inequalities. Collect. Math. 54, 181-216 (2003)

24. Gogatishvili, A, Stepanov, VD: Reduction theorems for weighted integral inequalities on the cone of monotone functions. Russ. Math. Surv. 68(4), 597-664 (2013)

25. Kantorovich, LV, Akilov, GP: Functional Analysis. Pergamon, Oxford (1982)

26. Sinnamon, G, Stepanov, VD: The weighted Hardy inequality: new proofs and the case $p=1$. J. Lond. Math. Soc. 54 89-101 (1996) 